\title{
Discrimination of Angle-Doppler Signatures using Arbitrary Phase Center Motion for MIMO Radars
}

\author{
Christian Hammes* ${ }^{\dagger}$, Yogesh Nijsure* ${ }^{*}$, Bhavani Shankar M. R.*, Udo Schröder ${ }^{\ddagger}$ and Björn Ottersten* \\ ${ }^{*}$ University of Luxembourg, SnT, ${ }^{\ddagger}$ IEE Contern, ${ }^{\dagger}$ Email: christian.hammes@uni.lu
}

\begin{abstract}
A novel Phase Center Motion (PCM) based technique for discriminating angle-Doppler signatures within Multiple-Input-Multiple-Output (MIMO) radars using Frequency Modulated Continuous Wave (FMCW) has been explored in this work. The PCM technique induces angle dependent Doppler shifts in the back-scattered signal, wherein a modified Doppler post processing for FMCW leads to joint angle-Doppler processing. Specifically, we intend to design unique spatialtemporal motion of the phase center on each individual MIMO radar channel in an effort to synthesize nearly orthogonal angleDoppler signatures. Subsequently, we also develop a MIMO radar receiver design, which would be capable of discriminating between these induced angle-Doppler signatures. The asymptotic investigation provides a Bessel function characteristic. Simulation results demonstrate a significant side-lobe suppression of $8.5 \mathrm{~dB}$ for an individual PCM trajectory and $7 \mathrm{~dB}$ over distinct PCM trajectories, in an attempt towards realization of nearly orthogonal MIMO radar channels.
\end{abstract}

\section{INTRODUCTION}

In conventional Synthetic Aperture Radars (SAR), the concept of Phase Center Motion (PCM) has been commonly referred to as the Displaced Phase Center Antenna (DPCA) technique, wherein the phase center over the radar antenna array is displaced in the opposite direction of the radar platform motion in an effort to keep the clutter statistically constant, which could be canceled subsequently from the radar back-scatter, as shown in works like [1], [2] and [3]. Works like [1] have used the aforementioned DPCA technique within airborne SAR radars. Specifically, an algorithm which uses the DPCA principle has been employed in order to reconstruct the linear sampling of the aperture.

Time division multiplex (TDM) Multiple-Input-MultipleOutput (MIMO) concept has been employed by activating a single pair of transmitter and receiver antenna element within the array to achieve orthogonality with respect to angle of arrival [4]. Within this approach, the authors have introduced different antenna switching schemes each yielding a single linear PCM design. These schemes exploit angle coding to achieve orthogonality that is necessary for the realization of the virtual MIMO approach. A virtual MIMO array is produced by the spatial convolution of the real transmit and receive antenna array which gives a much larger array while physically using smaller array size [5].

Time modulated arrays have been investigated in works like [6]-[10] with an objective of suppressing the side-lobe levels within the radiation beam pattern of the radar transmitter. Works like [6] and [7] have presented the bidirectional and unidirectional PCM approaches respectively for the uniform linear array. In [8], an algorithm has been presented which uses optimization techniques in order to determine the amplitudes of the signal over a time modulated linear antenna array. Pulse shifting techniques within a time modulated array have been shown in [9]. The authors in [10] have been presented a study on FMCW pulses in time modulated arrays.

Works on time modulated arrays are equivalent to linear PCM approaches [6]-[10]. In contrast to the prior-art on linear PCM for obtaining low side lobe level, we introduce the concept of PCM trajectory, which describes the spatial-temporal motion of the phase center within the array structure. The current work addresses arbitrary nonlinear PCM trajectories in order to enhance angle-Doppler discrimination by suppressing the angle-Doppler coupling problem [4]. Moreover, multiple PCM trajectory design has not been well investigated within the existing literature; consequently in this work we intend to design and exploit multiple PCM trajectories in order to achieve diversity over the target back-scatter to realize a MIMO radar set-up.

The contributions of the proposed work include:

- Enhanced unambiguous target discrimination using virtual MIMO and an arbitrary PCM trajectory based signal design.

- Design of a correlation based PCM trajectory filter at the receiver in addition to the conventional range-Doppler processing at the FMCW radar receiver. The same has been demonstrated using the sinusoidal PCM trajectory.

- Realization of the MIMO radar paradigm by synthesizing multiple PCM trajectories which induce nearly orthogonal angle-Doppler signatures.

- For the sinusoidal PCM, analytical characterization of the ambiguity function is derived under the assumption of large number of chirps within a coherent processing interval. This characterization highlights various properties of the sinusoidal PCM including side-lobe suppression.

In Section II, the signal model is introduced. Section III describes and elaborates the proposed method with reference to sinusoidal PCM as a special case and its asymptotic characterization. Simulation results with regards to the angleDoppler discrimination and the achievable orthogonality between distinct MIMO channels through use of the proposed PCM trajectory design has been presented in Section IV. Section $\mathrm{V}$ contains the conclusion and remarks for the full paper extension.

Throughout the work we use, $\|\cdot\|$ which denotes the $l_{2}$-norm. $[\cdot]_{\eta, \gamma}$ defines a matrix entry with row index $\eta$ and column 


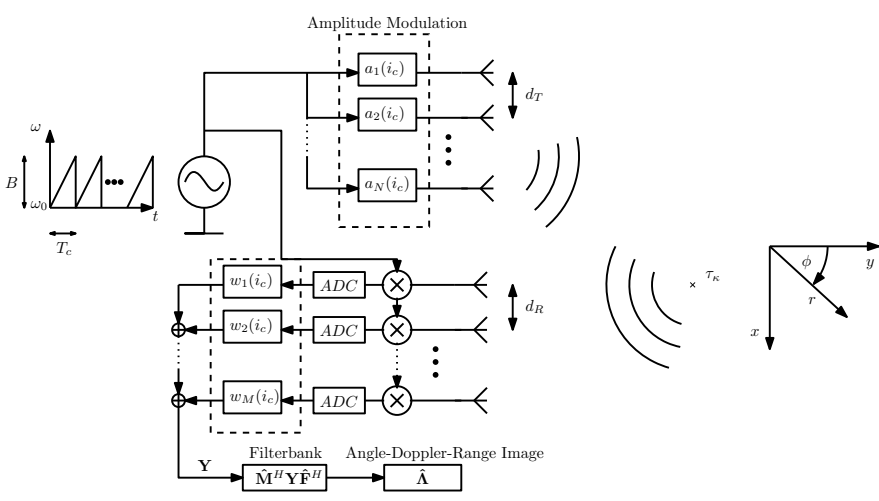

Fig. 1. System diagram

index $\gamma$. The notation $[\cdot]_{\eta}$ indicates a column vector element with the index $\eta$. The transpose is denoted as $(\cdot)^{T}$ and the conjugate transpose is denoted as $(\cdot)^{H}$, respectively. The arg operator provides the angle between the real and imaginary part of a complex number. The symbols $\mathbb{C}, \mathbb{Z}$ and $\mathbb{R}$ define the set of complex, integer and real numbers.

\section{System Model for Phase Center Motion}

Any radiating object at a large distance to the observer is seen by the latter as a point source. The origin of such a point source is called phase center [11]. The PCM approach exploits the effect of a phase trajectory or variations induced in the position of the phase center by appropriate signal design. The target motion and its angular positions then transform this trajectory. Appropriate receiver processing extracts unambiguous target angle-Doppler signatures from the transformed phase center trajectory.

Figure 1 depicts a conventional Frequency Modulated Continuous Wave (FMCW) system used for the PCM approach, where chirp sequences with the carrier frequency $f_{0}$ together with FMCW modulator bandwidth $B$ and chirp duration $T_{c}$ are used [14]. The $N$ transmit antenna elements are mounted on a uniform linear array along the $x$-direction with a transmit antenna inter-element spacing $d_{T}$, while the $M$ receive antenna elements are mounted on a uniform linear array along the $x$ direction with a receive antenna inter-element spacing $d_{R}$. The PCM is enabled within the array structure by an appropriate choice of transmitter Amplitude Modulation (AM) weights and receiver coefficients. We further assume that these coefficients are constant over a chirp, but vary across chirps. This translates into a phase center which is constant within the chirps but varies across them. Letting $i_{c}$ to be the chirp index, the corresponding AM weights are denoted by $a_{n}\left(i_{c}\right)$ for the $n$ th element and $w_{m}\left(i_{c}\right)$ denotes the receiver weighting for the $m$-th element. This is clearly depicted in figure 1 . Due to the PCM, a Doppler shift in addition to the target Doppler shift appears which necessitates a different transformation for processing other than the conventional Fast Fourier Transform (FFT) [14]. Therefore, the modified transformation, which is illustrated in Figure 1 as the matrix $\hat{\mathbf{M}}$, is another contribution of this paper. Since the matrix $\hat{M}$ is a function of the PCM trajectory, the matrix represents a trajectory matched filterbank in order to facilitate a two dimensional target angleDoppler image.

\section{A. System Model}

The signal vector at the output of the transmit antenna array during the time instance $t$ of the chirp $i_{c}$, denoted by $\mathbf{u}_{i_{c}}(t) \in$ $\mathbb{C}^{N \times 1}$, is the product of the Frequency Modulated Continuous Wave (FMCW) modulator and the transmit AM vector $\mathbf{a}\left(i_{c}\right)=$ $\left[a_{1}\left(i_{c}\right) \cdots a_{N}\left(i_{c}\right)\right]^{T} \in \mathbb{C}^{N \times 1}$,

$$
\mathbf{u}_{i_{c}}(t)=\mathbf{a}\left(i_{c}\right) \exp \left(j\left(\omega_{0} t+\frac{B}{T_{c}} t^{2}\right)\right) .
$$

In (1), $\omega_{0}=2 \pi f_{0}$ denotes the carrier angular frequency, $B$ is the FMCW bandwidth, $T_{c}$ is the sweep duration and $j=$ $\sqrt{-1}$. Subsequently, the $M \times N$ signal propagation paths with respect to the $\kappa$-th target can be described by the channel matrix $\mathbf{S}_{\kappa} \in \mathbb{C}^{M \times N}$ and time varying scalar $s_{\kappa}\left(i_{c}\right)$. A detailed target model is described in Section II-B. Similar to [4], the paper focuses on an ideal case where the receiver noise is neglected in the derivation. A sensitivity analysis with regards to noise is investigated numerically in Section IV. The receive signal vector $\mathbf{v}_{i_{c}}(t) \in \mathbb{C}^{M \times 1}$ takes the form,

$$
\mathbf{v}_{i_{c}}(t)=\sum_{\kappa=1}^{K} s_{\kappa}(t) \mathbf{S}_{\kappa} \mathbf{u}_{i_{c}}\left(t-t_{\kappa}\right) .
$$

The signal round trip delay for the $\kappa$-th target is defined as $t_{\kappa}=\frac{2 r_{\kappa}}{c_{0}}$, where $r_{\kappa}$ is the radial distance as shown in Section II-B and $c_{0}$ denotes the speed of light. The signal $\mathbf{v}_{i_{c}}(t)$ is down-mixed with the local oscillator and discretised with the sampling time $T_{s}$. The resulting samples are weighted by the time dependent weight vector $\mathbf{w}\left(i_{c}\right) \in \mathbb{C}^{M \times 1}$. In addition to the phase center, the target position is also assumed to be constant within one chirp and remains in one range resolution bin within the coherent processing interval.

The receive signal for $\mathcal{I}_{c}$ chirps, sampled at $\mathcal{I}_{s}$ samples per chirp, can be compactly expressed as a $\mathcal{I}_{c} \times \mathcal{I}_{s}$ matrix $\mathbf{Y} \in \mathbb{C}^{\mathcal{I}_{c} \times \mathcal{I}_{s}}$, where the matrix entries are $[\mathbf{Y}]_{i_{c}, i_{s}}=$ $\mathbf{w}^{T}\left(i_{c}\right) \mathbf{v}_{i_{c}}\left(i_{s} T_{s}\right) \exp \left(-j\left(\omega_{0} i_{s} T_{s}+\frac{B}{T_{c}}\left(i_{s} T_{s}\right)^{2}\right)\right)$. The matrix $\mathbf{Y}$ can be expressed in terms of a range vector $\boldsymbol{\mu}_{\kappa} \in \mathbb{C}^{\mathcal{I}_{s} \times 1}$ and Doppler vector $\boldsymbol{\nu}_{\kappa} \in \mathbb{C}^{\mathcal{I}_{c} \times 1}$ with the entries $\left[\boldsymbol{\mu}_{\kappa}\right]_{i_{s}}=$ $\exp \left(-j \rho_{\kappa} T_{s} i_{s}\right)$ and $\left[\boldsymbol{\nu}_{\kappa}\right]_{i_{c}}=c_{\kappa} s_{\kappa}\left(i_{c}\right) \boldsymbol{w}^{T}\left(i_{c}\right) \mathbf{S}_{\kappa} \mathbf{a}\left(i_{c}\right)$ as,

$$
\mathbf{Y}=\sum_{\kappa=1}^{K} \boldsymbol{\nu}_{\kappa} \boldsymbol{\mu}_{\kappa}^{T}
$$

The complex constant $c_{\kappa}=\exp \left(j\left(\omega_{0} t_{\kappa}+\frac{B}{T_{c}} t_{\kappa}^{2}\right)\right)$ is a result of the FMCW down-mixing and the angular frequency corresponding to the range information is denoted as $\rho_{\kappa}=4 \frac{B}{T_{c}} \frac{r_{\kappa}}{c_{0}}$. Referring to (3), the range information is separable from PCM and Doppler information.

\section{B. Target Model}

The target as well as the antenna elements are assumed to be point-like isotropic radiators. For a considered MIMO configuration, there are $N \times M$ propagation paths. The distance $d_{m n}^{\kappa}$ between the $n$-th transmit element and the $m$-th receive element is defined as the distance from $n$-th transmit element 


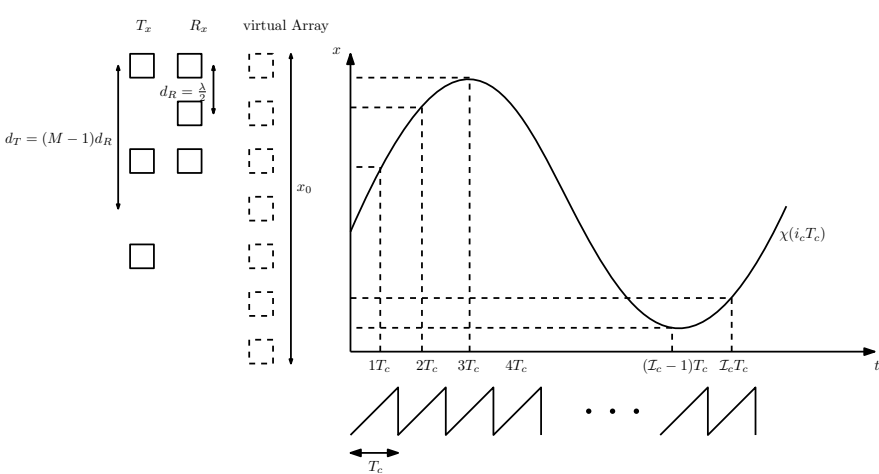

Fig. 2. Virtual MIMO PCM for sinusoidal trajectory

to the $\kappa$-th target plus the distance from the $\kappa$-th target to the $m$-th receive antenna element [12] and takes the form,

$$
\begin{aligned}
d_{m n}^{\kappa} & =\left\|\mathbf{x}_{\kappa}-\mathbf{x}_{m}\right\|+\left\|\mathbf{x}_{\kappa}-\mathbf{x}_{n}\right\| \\
& \approx 2 r_{\kappa}+\left((n-1) d_{T}+(m-1) d_{R}\right) \sin \left(\phi_{\kappa}\right),
\end{aligned}
$$

where $\mathbf{x}_{\kappa}$ is the $\kappa$-th target position in Cartesian coordinates, $\mathbf{x}_{n}$ and $\mathbf{x}_{m}$ are the positions of the transmit and receive antenna elements in Cartesian coordinates, $r_{\kappa}$ and $\phi_{\kappa}$ denotes the $\kappa$-th target position in spherical coordinates. The first antenna element is assumed to be the origin of both coordinate systems as depicted in Figure 2. The distance $d_{m n}^{\kappa}$, when multiplied by the free space wave number $k_{0}$, provides the phase information. The wavelength $\lambda$ corresponds to the free space wave number $k_{0}=\frac{2 \pi}{\lambda}$ which then again corresponds to speed of light and the carrier frequency $f_{0}=\frac{c_{0}}{\lambda}$. Using (4), the phase information can be split into a radial $2 k_{0} r_{\kappa}$ and angular phase term $\varphi_{m n}^{\kappa}=k_{0} d_{m n}^{\kappa}=k_{0} \sin \left(\phi_{\kappa}\right)\left((n-1) d_{T}+(m-1) d_{R}\right)$. The target radar cross section $\sigma_{\kappa}$ together with the target Doppler shift $\delta_{\kappa}$ and radial phase term constitute the time varying scalar $s_{\kappa}\left(i_{c}\right)$, which can be presented as [12],

$$
s_{\kappa}\left(i_{c}\right)=\sigma_{\kappa} \exp \left(j \delta_{\kappa} T_{c} i_{c}\right) .
$$

The $M \times N$ MIMO propagation channel matrix $\mathbf{S}_{\kappa} \in \mathbb{C}^{M \times N}$ is obtained as

$$
\left[\mathbf{S}_{\kappa}\right]_{m, n}=\exp \left(j \varphi_{m n}^{\kappa}\right)
$$

\section{Proposed Method And Sinusoidal PCM}

The proposed method is based on a virtual MIMO paradigm which is obtained by the spatial convolution of the sparse transmit array with a filled receiver array as depicted in Figure 2. In particular, the proposed method uses a novel choice of $\mathrm{AM}$ weights and receiver processing to enable and benefit from a continuous PCM within the virtual array.

\section{A. Phase Center Motion Framework}

The MIMO channel matrix $\mathbf{S}_{\kappa}$, together with the transmit and receive weight vectors $\mathbf{a}\left(i_{c}\right)$ and $\mathbf{w}\left(i_{c}\right)$ contain the phase center trajectory information. Let $k_{\kappa}^{x}=k_{0} \sin \left(\phi_{\kappa}\right)$ denotes the propagation vector for the $\kappa$-th target in the $x$-direction. The composite phase center $\chi\left(i_{c}\right)$, as seen at the receiver, is defined as the group delay of transmit and receive array group factor. If the weighting vectors $\mathbf{w}\left(i_{c}\right)$ and $\mathbf{a}\left(i_{c}\right)$ fit the function of a fractional delay filter [13], like a truncated sincfunction, the phase center $\chi\left(i_{c}\right)$ can take any position within the array structure to a good approximation. Since the matrix $\mathbf{S}_{\kappa}$ is separable into transmit and receive components, the phase center $\chi\left(i_{c}\right)$ is the superposition of the transmit $\alpha\left(i_{c}\right)$ and receive $\beta\left(i_{c}\right)$ phase center which enables the application of virtual MIMO concept as shown in Section III-B,

$$
\begin{aligned}
\chi\left(i_{c}\right) & =\frac{\partial}{\partial k_{\kappa}^{x}} \arg \left(\mathbf{w}^{T}\left(i_{c}\right) \mathbf{S}_{\kappa} \mathbf{a}\left(i_{c}\right)\right) \\
& \approx \frac{\partial}{\partial k_{\kappa}^{x}} \arg \left(e^{j k_{\kappa}^{x}\left(\alpha\left(i_{c}\right)+\beta\left(i_{c}\right)\right)}\right)=\alpha\left(i_{c}\right)+\beta\left(i_{c}\right) .
\end{aligned}
$$

If the weighting of transmit and receive array yields a pure phase center motion without beam pattern shaping, the Doppler vector entries can be simpified as $\left[\boldsymbol{\nu}_{\kappa}\right]_{i_{c}}=$ $\sigma_{\kappa} c_{\kappa} \exp \left(j\left(\delta_{\kappa} T_{c} i_{c}+k_{\kappa}^{x} \chi\left(i_{c}\right)\right)\right)$ and (3) simplifies to,

$$
\mathbf{Y}=\mathbf{M} \Lambda \mathbf{F} \text {. }
$$

The unique matrix $\mathbf{M} \in \mathbb{C}^{\mathcal{I}_{c} \times K}$ with the entries $[\mathbf{M}]_{i_{c}, \kappa}=$ $\frac{1}{\sqrt{\mathcal{I}_{c}}} \exp \left(j\left(\delta_{\kappa} T_{c} i_{c}+k_{\kappa}^{x} \chi\left(i_{c}\right)\right)\right)$ spans the column space of $\mathbf{Y}$, which contains the target angle and Doppler information as well as the phase center trajectory. The unique matrix $\mathbf{F} \in \mathbb{C}^{K \times \mathcal{I}_{s}}$ with the entries $[\mathbf{F}]_{\kappa, i_{s}}=\frac{1}{\sqrt{\mathcal{I}_{s}}} \exp \left(j \rho_{\kappa} T_{s} i_{s}\right)$ spans the row space of $\mathbf{Y}$ which contains the target range information. The diagonal matrix $\boldsymbol{\Lambda}=\sqrt{\mathcal{I}_{c} \mathcal{I}_{s}} \operatorname{diag}\left(c_{\kappa} \sigma_{\kappa}\right) \in \mathbb{C}^{K \times K}$ represents the energy from the $\kappa$-th target.

Therefore, the matrix $\mathbf{M}$ is parametrized by the target Doppler shifts $\delta_{\kappa}$ and target angular position $\phi_{\kappa}$ or $k_{\kappa}^{x}$, respectively. The resolution with regards to Doppler is limited by the inverse coherent processing interval $\Delta_{\delta}=\frac{2 \pi}{\mathcal{I}_{c} T_{c}}$ [12] and the angular resolution has the limit of the inverse virtual array size $\Delta_{\phi}=\frac{4 \pi}{(N(M-1)+1) \lambda}$ [5]. A grid for angle and Doppler with $\mathcal{I}_{c} \times(N(M-1)+1)$ bins can be defined based on the resolution limits. Therefore, the index $q \in$ $\left[1 \cdots \mathcal{I}_{c}\right]$ represents the Doppler dimension and the index $p \in[1 \cdots(M-1) N+1]$ the angular dimension. The resolution of the range, associated with the angular frequency, is limited by the chirp time $\Delta_{\rho}=\frac{2 \pi}{T_{c}}$. The index $l \in\left[1 \cdots \mathcal{I}_{s}\right]$ corresponds with the range dimension. A target can only be resolved by the resolution limits and the total number of $K$ targets and their positions are unknown. The trajectory matched filter matrices $\hat{\mathbf{M}} \in \mathbb{C}^{\mathcal{I}_{c} \times \mathcal{I}_{c}(N(M-1)+1)}$ with the entries $[\hat{\mathbf{M}}]_{\left(p \mathcal{I}_{c}+q\right), i_{c}}=\frac{1}{\mathcal{I}_{c}} \exp \left(j\left((q-1) \Delta_{\delta} T_{c} i_{c}+(p-\right.\right.$ 1) $\left.\left.\Delta_{\phi} \chi\left(i_{c}\right)\right)\right)$ and $\hat{\mathbf{F}} \in \mathbb{C}^{\mathcal{I}_{s} \times \mathcal{I}_{s}}$ with the entries $[\hat{\mathbf{F}}]_{l, i_{s}}=$ $\frac{1}{\mathcal{I}_{s}} \exp \left(j(l-1) \Delta_{\rho} T_{s} i_{s}\right)$ are designed in an effort to cover all possible combinations within the resolution limits. The filterbank output $\hat{\Lambda} \in \mathbb{C}^{\mathcal{I}_{c}(N(M-1)+1) \times \mathcal{I}_{s}}$ contains the elements of an angle-Doppler-Range plot,

$$
\hat{\mathbf{\Lambda}}=\hat{\mathbf{M}}^{H} \mathbf{Y} \hat{\mathbf{F}}^{H} \text {. }
$$

The proposed method is to design the phase center trajectory $\chi\left(i_{c}\right)$ such that each row of $\hat{\mathbf{M}}$ is nearly orthogonal to each other. The target discrimination with regards to angle-Doppler then becomes enhanced. Since range and Doppler processing are separable, the next section just deals with the design of $\hat{\mathbf{M}}$. In case of multiple trajectories, there are multiple $\hat{\mathbf{M}}$, which is discussed in the next section for sinusoidal trajectories. 


\section{B. Sinusoidal Phase Center Motion using Virtually Filled} Arrays

In order to design a matrix $\hat{\mathbf{M}}$ that is, to a good approximation row orthogonal, the PCM is adjusted to a sinusoidal trajectory $\chi\left(i_{c}\right)=\frac{x_{0}}{2} \sin \left(\frac{2 \pi}{\mathcal{I}_{c}} i_{c}\right)$, where $x_{0}=(N(M-1)+1) \frac{\lambda}{2}$ is the total array size. The AM is assumed to be binary wherein the transmit antenna elements can either be switched on or off. As a consequence of the binary AM condition and the arrangement of transmit antenna elements as illustrated in Figure 1, the transmit phase center is restricted to discrete values with the inter-element spacing, such that the transmit phase center $\alpha\left(i_{c}\right)=\epsilon\left(i_{c}\right)(M-1) d_{R}$ for $\epsilon \in \mathbb{Z}$ where $0 \leq \epsilon \leq N-1$. Therefore, the transmit phase center $\alpha\left(i_{c}\right)$ is restricted to discrete values, which is a reasonable assumption for practical applications. The receive processing is performed in the digital domain and therefore the receive weight vector $\mathbf{w}\left(i_{c}\right)$ can be easily adjusted to the coefficients of a fractional delay filter. However, for the sinusoidal example, a truncated sinc-function is used for receive PCM, thus the weights are restricted to real values between minus one and one $\mathbf{w}\left(i_{c}\right) \in \mathbb{R}^{M \times 1}$ where $-1 \leq[w]_{\zeta} \leq 1$. The receive phase center position, $\beta\left(i_{c}\right)$ is bounded by the receive array size, but it can take on every real value within these bounds $\beta\left(i_{c}\right) \in \mathbb{R}$ where $0 \leq \beta\left(i_{c}\right) \leq(M-1) \frac{\lambda}{2}$ by fitting a sinc-function, where the function maximum lies within the receive array bounds. The superposition of receive and transmit phase center enables the total phase center $\chi\left(i_{c}\right)=\alpha\left(i_{c}\right)+\beta\left(i_{c}\right)$ to take on every value within sparse array structure in Figure 2 even though the antenna element arrangement is sparse,

$$
\begin{aligned}
{[\mathbf{w}]_{m}\left(i_{c}\right) } & =\operatorname{sinc}\left(m-\frac{\beta\left(i_{c}\right)}{d_{R}}\right) \\
& =\operatorname{sinc}\left(m-\frac{\chi\left(i_{c}\right)-\alpha\left(i_{c}\right)}{d_{R}}\right)
\end{aligned}
$$

The array appears to be filled and therefore the virtual MIMO concept is enabled. Furthermore, the sinusoidal PCM and the corresponding transmit $\mathrm{AM}$ vector $\mathbf{a}\left(i_{c}\right)$ and the receive weighting vector $\mathbf{w}\left(i_{c}\right)$ are completely determined by the assumptions made in this section, like binary AM, real receive weights and array structure.

\section{Asymptotic Bessel Function Characteristic for Sinusoidal $P C M$}

The asymptotic consideration of sinusoidal PCM yields a closed form ambiguity function expression for analytical investigation. The trajectory matched filter output can be analytically investigated with respect to target discrimination and the associated resolution and side lobe level. Furthermore, asymptotic formulas for sinusoidal PCM are providing an analyzing tool for orthogonality with respect to multiple PCM trajectories.

In order to derive the asymptotic formula, equation (9) can be separated using (3). Therefore, the angle-Doppler trajectory matched filter output $\tilde{\mathbf{j}} \in \mathbb{C}^{\mathcal{I}_{c}(N(M-1)+1) \times 1}$ can be formulated as,

$$
\begin{aligned}
{[\tilde{\mathbf{j}}]_{\left(p \mathcal{I}_{c}+q\right)} } & =\left[\sum_{\kappa=1}^{K} \hat{\mathbf{M}} \boldsymbol{\nu}_{\kappa}\right]_{\left(p \mathcal{I}_{c}+q\right)} \\
& =\sum_{\kappa=1}^{K} \frac{c_{\kappa} \sigma_{\kappa}}{\mathcal{I}_{c}} \sum_{i_{c}=0}^{\mathcal{I}_{c}} \exp \left(j \left(\left((q-1) \Delta_{\delta}-\delta_{\kappa}\right) T_{c} i_{c}\right.\right. \\
& \left.\left.+\left((p-1) \Delta_{\phi}-k_{\kappa}^{x}\right) \chi\left(i_{c}\right)\right)\right)
\end{aligned}
$$

For the sake of illustration, the vector $\tilde{\mathbf{j}}$ is rearranged to a matrix $\tilde{\mathbf{J}} \in \mathbb{C}^{\mathcal{I}_{c} \times N(M-1)+1}$, where the column space represents the Doppler domain and the row space represents the angular domain. Using the aforementioned matrix notation and substituting $\epsilon(q)=\left((q-1) \Delta_{\delta}-\delta_{\kappa}\right) \frac{T_{c} \mathcal{I}_{c}}{2 \pi}$ and $\gamma(p)=$ $\frac{x_{0}}{2}\left((p-1) \Delta_{\phi}-k_{\kappa}^{x}\right)$, equation (11) can be written as follows by inserting the sinusoidal PCM, where the trajectory angular frequency is defined as $\omega_{T}=\frac{2 \pi}{\mathcal{I}_{c} T_{c}}$,

$$
\begin{aligned}
& {[\tilde{\mathbf{J}}]_{p, q}=\sum_{\kappa=1}^{K} \frac{c_{\kappa} \sigma_{\kappa}}{\mathcal{I}_{c} T_{c}}} \\
& \times \sum_{i_{c}=0}^{\mathcal{I}_{c}} \exp \left(j\left(\epsilon(q) \omega_{T} i_{c} T_{c}+\gamma(p) \sin \left(\omega_{T} i_{c} T_{c}\right)\right)\right) T_{c} .
\end{aligned}
$$

The coherent processing interval can be defined as $T_{C P I}=T_{c} \mathcal{I}_{c}$. Further, the definition $\hat{J}_{\kappa}(\epsilon(q), \gamma(p))=$ $\frac{1}{T_{C P I}} \sum_{i_{c}=0}^{\mathcal{I}_{c}} \exp \left(j\left(\epsilon(q) \omega_{T} T_{c} i_{c}+\gamma(p) \sin \left(\omega_{T} i_{c} T_{c}\right)\right)\right) T_{c}$ yields,

$$
[\tilde{\mathbf{J}}]_{p, q}=\sum_{\kappa=1}^{K} c_{\kappa} \sigma_{\kappa} \hat{J}_{\kappa}(\epsilon(q), \gamma(p)) .
$$

The asymptotic assumption is that the chirp duration goes to zero $T_{c} \rightarrow 0$, while the coherent processing interval keeps constant $T_{C P I}=$ const,

$$
\begin{aligned}
& J_{\kappa}(\epsilon(q), \gamma(p))=\lim _{T_{c} \rightarrow 0} \hat{J}_{\kappa}(\epsilon(q), \gamma(p)) \\
& =\int_{0}^{T_{C P I}} \frac{\exp \left(j\left(\epsilon(q) \frac{2 \pi}{T_{C P I}} t+\gamma(p) \sin \left(\frac{2 \pi}{T_{C P I}} t\right)\right)\right)}{T_{C P I}} d t .
\end{aligned}
$$

Equation (14) yields a Bessel function of the first kind as the asymptotic result for the $\kappa$-th target. The asymptotic trajectory matched filter output $\mathbf{J}$ is the superposition of shifted first kind Bessel functions,

$$
[\mathbf{J}]_{p, q}=\lim _{T_{c} \rightarrow 0}[\tilde{\mathbf{J}}]_{p, q}=\sum_{\kappa=1}^{K} c_{\kappa} \sigma_{\kappa} J_{\kappa}(\epsilon(q), \gamma(p)) .
$$

The squared $L_{2}$-norm of equation (15) leads to the angleDoppler ambiguity function. Targets in different resolutions bin can be seen as independent, therefore the squared matched filter output can be well approximated by the superposition of single target responses,

$$
\left\|[\mathbf{J}]_{p, q}\right\|^{2} \approx \sum_{\kappa=1}^{K}\left\|c_{\kappa} \sigma_{\kappa} J_{\kappa}(\epsilon(q), \gamma(p))\right\|^{2} .
$$

Another important aspect is the orthogonal property of Bessel function with respect to angular frequency $\omega_{T}$. In terms of PCM does it mean, that the sinusoidal trajectory has an integer 

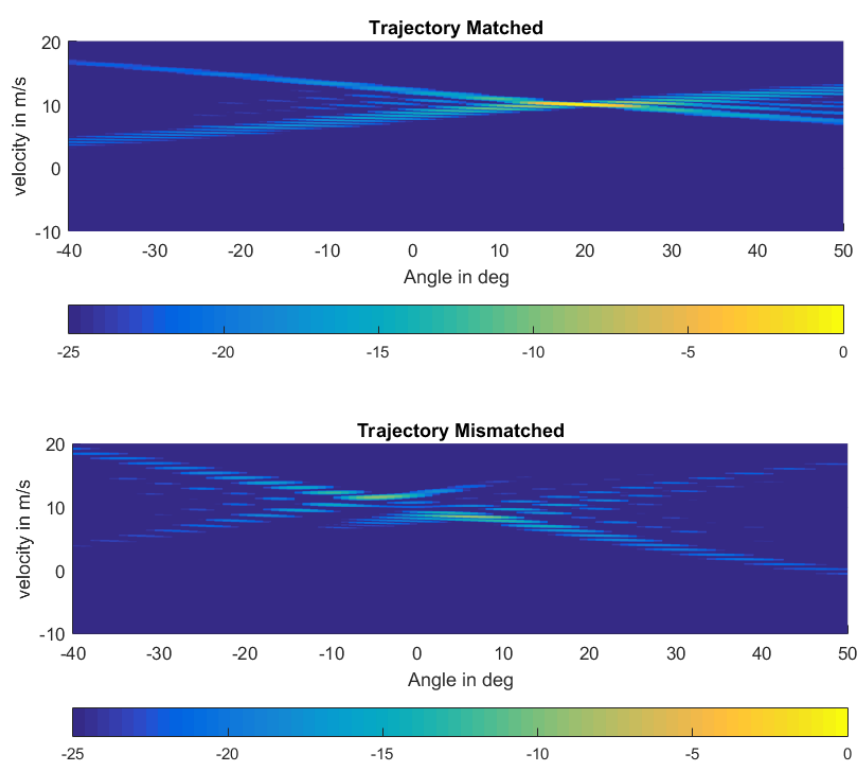

Fig. 3. Upper Plot: Angle-Doppler ambiguity function for sinusoidal trajectory with a single target located at angle $\phi_{1}=20^{\circ}$ and velocity $v_{1}=10 \mathrm{~m} / \mathrm{s}$ with a Side Lobe Level of $-8.5 \mathrm{~dB}$ Lower Plot: Filter bank output for trajectory mismatched with respect to angular frequency $\omega_{T}$ of the sinusoidal PCM; signal suppression of $7 \mathrm{~dB}$

multiple of the angular frequency $\omega_{T}$. The orthogonality property just holds for Bessel functions of the same order, therefore sinusoidal trajectories with different velocities are only nearly orthogonal. The asymptotic Bessel function properties are confirmed by simulation results.

\section{Simulation Results}

The simulation is carried out with an array configuration as depicted in Figure 2 with $N=4$ transmit and $M=4$ receive antennas. The carrier frequency $f_{0}$ is set to $77 \mathrm{GHz}$. The total number of chirps is $\mathcal{I}_{c}=512$ with a chirp duration of $T_{c}=10 \mu \mathrm{s}$. The FMCW angular bandwidth $B$ is set to $4 \pi \mathrm{GHz}$. The sampling time is $T_{s}=\frac{4 \pi}{B}$ with a total intra chirp sampling number of $\mathcal{I}_{s}=10000$. While the first target is located at the angle $\phi_{1}=20^{\circ}$, range $r_{1}=10 \mathrm{~m}$ and has the velocity of $v_{1}=10 \mathrm{~m} / \mathrm{s}$, the second target is located at the angle $\phi_{2}=-5^{\circ}$, range $r_{2}=10 \mathrm{~m}$ and has the velocity of $v_{2}=0 \mathrm{~m} / \mathrm{s}$. The signal to noise ratio is set to $S N R=-10 \mathrm{~dB}$.

The upper plot in Figure 3 shows the ambiguity function for an angle-Doppler plot in $\mathrm{dB}$-scale. A sharp peak appears at the target location in this plane, which enables an unambiguous angle-Doppler parameter estimation. The Side Lobe Level $(S L L=-8.5 \mathrm{~dB})$ is a promising result demonstrated by our proposed PCM signal design.

While the angle-Doppler ambiguity function result showed the potential of a single trajectory, we now consider multiple trajectories obtained by superposition of sinusoidal PCM trajectories. The component trajectories have different angular frequencies, which can be chosen towards obtaining orthogonality in MIMO radars. In particular, the lower plot in Figure 3 illustrates the output of a mismatched trajectory filter. The filter trajectory has twice the angular frequency of the input
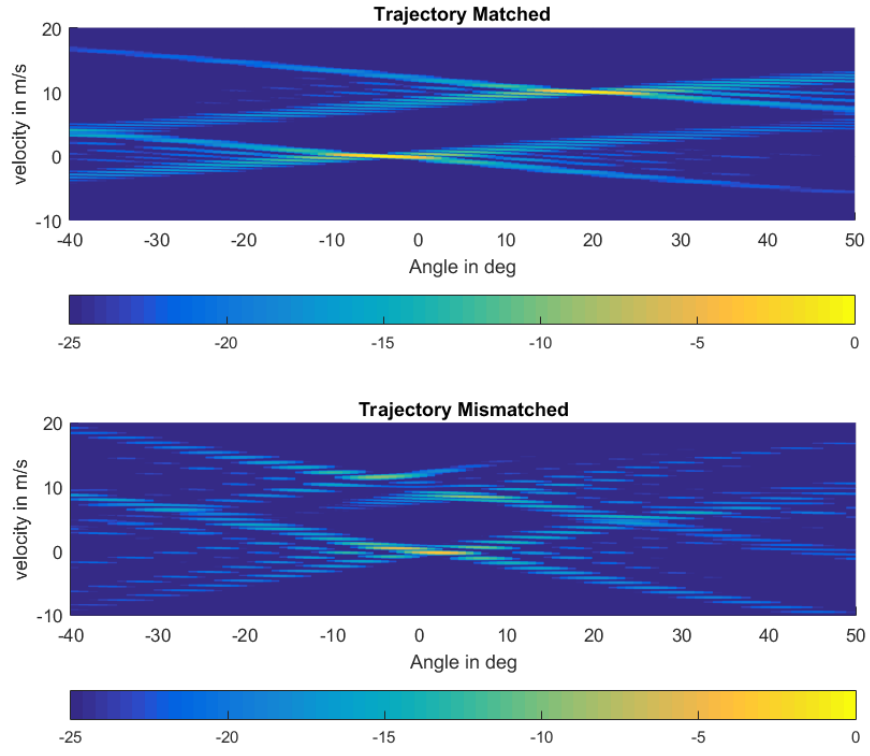

Fig. 4. Upper Plot: Angle-Doppler ambiguity function for sinusoidal trajectory with two targets, one located at angle $\phi_{1}=20^{\circ}$ and velocity $v_{1}=10 \mathrm{~m} / \mathrm{s}$ and the other at angle $\phi_{2}=-5^{\circ}$ and velocity $v_{5}=0 \mathrm{~m} / \mathrm{s}$ Lower Plot: Filter bank output for trajectory mismatched with respect to angular frequency $\omega_{T}$ of the sinusoidal PCM for the two target case

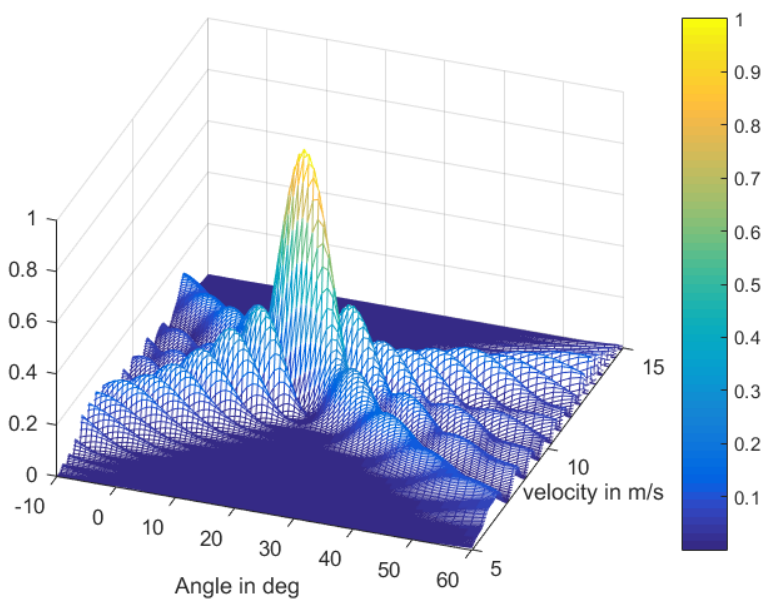

Fig. 5. Ambiguity function and its asymptotic Bessel characteristic for a single target located at angle $\phi_{1}=20^{\circ}$ and velocity $v_{1}=10 \mathrm{~m} / \mathrm{s}$

trajectory and therefore mismatched in terms of asymptotic Bessel considerations. The input signal is suppressed by $7 \mathrm{~dB}$, which indicates approximate orthogonality of the trajectories and hence independent processing of related signals. Thus the trajectory approach enables a framework for exploiting a new degree of freedom towards achieving orthogonality in MIMO radars.

Figure 4 illustrates the two target case matched (upper plot figure 4) and mismatched (lower plot figure 4) sinusoidal trajectory. The appropriate target peak and its side-lobes appear as a superposition of single target ambiguity functions and therefore the two target case confirms the superposition principle in (16). The trajectory mismatched filter output performance is similar to the single target case with regards to the input signal suppression of approximately $7 \mathrm{~dB}$. 
Figure 5 depicts a sector of figure 3 in linear scale and in three dimensional view. As derived in subsection III-C, the ambiguity function asymptotes has a Bessel function characteristic where the angular domain represents the argument and the velocity domain represents the order of first kind Bessel functions. Figure 5 illustrates clearly the Bessel shape and the symmetry properties. Therefore, the simulation result confirms the asymptotic behavior.

Furthermore, the trajectory matched filter approach provides good properties with regards to noise suppression. The rather low SNR has almost no influence on the ambiguity function for the chosen set of parameters.

\section{CONCLUSION}

This work proposes a novel radar waveform design approach based on arbitrary PCM trajectories within MIMO radar paradigm. The primary objective of this design approach is to achieve Thumbtack like response of MIMO radar ambiguity function in addition to accomplishing orthogonality over distinct MIMO radar channels. The attractiveness of the approach is exemplified by the design of sinusoidal trajectories which exhibits an asymptotic Bessel behavior for the angleDoppler ambiguity function. This behavior allows for wellknown characterization of the ambiguity function allowing precise evaluation of side-lobe levels and corroborating orthogonality. The attractiveness of this approach, in addition to unambiguous target discrimination and enableing of nearly orthogonal MIMO channels, lies in the linear matched filtering at the receiver which leads to fairly good noise suppression.

\section{ACKNOWLEDGMENT}

The authors would like to thank Dr. Thiemo Spielmann, IEE Contern, for his support. This work was supported by the National Research Fund, Luxembourg under AFR grant for Ph.D. project (Reference 11274469) on Enhancing Angular Resolution in Radar Through Dynamic Beam Steering and MIMO.

\section{REFERENCES}

[1] G. Krieger, N. Gebert, A. Moreira, "Unambiguous SAR Signal Reconstruction From Nonuniform Displaced Phase Center Sampling," IEEE Geosci. Remote Sens. Lett., vol. 1, no. 4, October 2004.

[2] D. Cerutti-Maori, I. Sikaneta, "A Generalization of DPCA Processing for Multichannel SAR/GMTI Radars," IEEE Trans. Geosci. Remote Sens., vol. 51, no. 1, January 2013.

[3] B. Dawidowicz, K. S. Kulpa, M. Malanowski, J. Misiurewicz, P. Samczynski, M. Smolarczyk, "DPCA Detection of Moving Targets in Airborne Passive Radar," IEEE Trans. Aerosp. Electron. Syst., vol. 48, no. 2, April 2012.

[4] D. Zoeke, A. Ziroff, "Phase Migration Effects in Moving Target Localization Using Switched MIMO Arrays," in Proceedings of the 12th European Radar Conference, September 2015.

[5] D. Bliss, K. Forsythe, G. Fawcett, "MIMO Radar: Resolution, Performance, and Waveforms," in Proceedings of ASAP, 2006.

[6] S. Yang, Y.-B. Gan, P. Khiang Tan, "Linear Antenna Arrays With Bidirectional Phase Center Motion," IEEE Trans. Antennas Propag., vol. 53, no. 5, April 2005.

[7] G. Li, S. Yang, Z. Nie, "Direction of Arrival Estimation in Time Modulated Linear Array With Unidirectional Phase Center Motion," IEEE Trans. Antennas Propag., vol. 58, no. 4, 2010.

[8] S. Yang, Y.-B. Gan, A. Qing, "Sideband Suppression in Time-Modulated Linear Arrays by the Differential Evolution Algorithm," IEEE Antennas Wireless Propag. Lett., vol. 1, 2002.
[9] L. Poli, P. Rocca, L. Manica, A. Massa, "Pattern synthesis in timemodulated linear array through pulse shifting," IET Microwave, Antennas and Propagation, February 2009.

[10] J. Guo, S. Yang, S.-W. Qu, Jun Hu, Zaiping Nie, "A Study on Linear Frequency Modulation Signal Transmission by 4-D Antenna Arrays," IEEE Trans. Antennas Propag., vol. 63, no. 12, December 2015.

[11] C. A. Balanis, "Antenna Theory- Analysis and Design, third edition," Wiley ISBN: 0-471-66782-X, 2005.

[12] P. Setlur and M. Rangaswamy, "Waveform Design for Radar STAP in Signal Dependent Interference," IEEE Trans. Signal Process., vol. 64, no. 1, January 2016.

[13] V. Välimäki and A. Haghparast, "Fractional Delay Filter Design Based on Truncated Lagrange Interpolation," IEEE Trans. Signal Process., vol. 64, no. 1, January 2016.

[14] S. Heuel, "Radar Waveform for A\&D and Automotive Radar," White Paper Rohde \& Schwarz, 2013 